45 USING SIMULATION TRAINING TO INTRODUCE MEDICAL STUDENTS TO COVID-19

C Rodrigues Cleto, L Boulstridge, C Stenhouse, D Craig. University Hospitals of Derby and Burton

\subsection{6/spcare-2021-PCC.63}

Introduction As the COVID-19 pandemic began to surge in the UK, medical students were removed from clinical placements. Consequently many medical students have had little direct experience of managing patients with COVID-19. This study reports, primarily, on a simulation training activity designed to introduce 3 rd year medical students to the communication challenges of treating patients with COVID19. It also reflects upon the benefits to students, of undertaking the role of a simulated patient during training activities.

Methods 21 third year medical students undertook teaching on breaking bad news. A modified SBAR model was taught as a consultation framework. Simulated scenarios, included two patients with COVID-19 and telephone consultations with the patients' families. Students wore surgical masks and undertook the role of doctor and patient or relative. Four students subsequently volunteered to act as simulated patients in training for new foundation doctors. Anonymised questionnaires, utilising ten-point visual analogue scales and qualitative questions, were used to evaluate the training.

Results $95 \%$ of students rated their course as excellent overall. High mean visual analogue scores and free-text responses revealed that the training had demonstrated the impact of COVID-19, PPE and restricted visiting on communication and clinical practice. It also informed students about the experiences of clinicians during the pandemic and provided a framework for their first attempts at breaking bad news. Acting as simulated patients was valuable for students' own training and encouraged them to consider the patients" perspectives.

Conclusions The COVID pandemic has had a significant impact upon medical education, causing concern about loss of clinical experience. Consequently a range of innovative teaching models have been reported on worldwide. This study suggests that simulation training can be used effectively to teach medical students about the challenges of clinical management, the complexities of communication and the impact on patients and doctors of COVID-19.

\section{SERVICE EVALUATION OF THE USE OF KETOROLAC AT THE MACMILLAN PALLIATIVE CARE UNIT (MPCU), SHEFFIELD 2018-2019}

Maimoona Ali, James Davies. Sheffield Teaching Hospitals NHS Foundation Trust

10.1136/spcare-2021-PCC.64

Background Ketorolac is used for pain control and also in pyrexia management at the end of life. It can be of significant benefit in severe pain related to cancer pain related to bone metastasis.

This service evaluation was to see the use of Ketorolac, to see whether it's being used with clear indications and what documented side effects and adverse outcomes, if any, there are in the patients we've used it in.

Methods Patient details who were prescribed Ketorolac were retrieved from a report run on the electronic prescribing system. Electronic patient records and if needed paper clinical notes were reviewed with blood results viewed online for data collection. Data was then analysed on Excel.

Results 16 patients were prescribed Ketorolac whilst as an inpatient at MPCU (11/07/2018-30/07/2019).

11 out of 16 patients (69\%) had their renal function checked prior to starting Ketorolac. 2 patients had is prescribed as a syringe driver, 7 patients had it prescribed regularly and 12 patients had it prescribed PRN.

Following administration of Ketorolac 9 patients did not have their renal function checked.

The most common reason documented for the bloods not been taken was the patient was for end of life care. Those patients when the renal function was checked there were no documented acute kidney injuries and their renal function was stable. No patient was discharged on Ketorolac.

Conclusions Patient centred care is a priority and the risk vs benefits of treatment options is challenging. This evaluation highlights that no patients had any documented acute kidney injuries who were prescribed Ketorolac. It will also enable the palliative medicine team to discuss with junior doctors, who regularly rotate, the importance of documenting decision making process which are individualised for our patients.

\section{EVALUATING THE IMPACT OF MEDICAL STUDENTS IN HOSPICE TEACHING WARD ROUNDS}

Bhajneek Grewal, Rosanna Hill, Suzie Gillon, Jason Ward, Clare Rayment. Leeds Teaching Hospitals NHS Trust, Bradford Teaching Hospitals NHS Foundation Trust, University of Leeds

\subsection{6/spcare-2021-PCC.65}

Introduction Healthcare professionals can be reluctant to invite medical students on ward rounds, especially in the context of emotive discussions. We present the results of a study analysing the impact of teaching ward rounds on patient experience in hospices.

Methods Eight hospices were invited to participate in this service evaluation. Six hospices across Yorkshire returned data. Inpatients were asked to complete an anonymous questionnaire after a teaching ward round, defined as consisting of a minimum of 3 members of staff, led by a senior doctor (consultant or specialty grade) and including at least one medical student.

Results Seventy-four questionnaires were returned. The vast majority (96\%) of patients were happy for students to be present on the ward round. None of the patients who were in a shared bay at the hospice felt that this prevented them from asking questions. The presence of a relative was felt to be a positive factor during the ward round experience. Only $11 \%$ of patients felt that the presence of students negatively affected the discussion of sensitive issues.

Conclusions Our study shows that most patients had a positive response to the presence of medical students on ward rounds. Overall teaching ward rounds did not appear to negatively impact upon the discussion of sensitive topics. This study contains a small sample size and the results cannot be generalised across a palliative care population. Nonetheless it provides data in support of medical students participating in teaching ward rounds in hospices where sensitive discussions frequently take place. 